\title{
FMCW Radar Prototype Development for Detection and Classification of Nano-Targets
}

\author{
Safiah Zulkifli and Alessio Balleri \\ Centre for Electronic Warfare, Information and Cyber \\ Cranfield University \\ Defence Academy of the UK \\ Shrivenham, SN6 8LA \\ \{Safiah.Binti-zulkifli, a.balleri\}@cranfield.ac.uk
}

\begin{abstract}
Detection and classification of nano-targets (less than $5 \mathrm{~cm}$ in size) are becoming important technical challenges as nano-targets are largely invisible to conventional radar. Nano-drones, for example, may soon become a tangible threat capable of providing short-range stealthy surveillance. Similarly, insect pests are posing a significant agricultural risk by causing crop losses and subsequently reducing the yields. Frequency Modulated Continuous Wave (FMCW) radar is a technology that can provide short-range detection, with no blind range and very high resolution, at a relatively low cost. This paper presents the latest results of an ongoing project aiming at designing and developing a low-cost and bespoke $24 \mathrm{GHz}$ FMCW radar prototype to enable detection of nano-targets and extract their Doppler signatures. A home-brew S-band FMCW radar prototype has been initially designed and developed, using off-the-shelf components, to demonstrate the feasibility of our proposed design solution and inform all future activities at $\mathbf{2 4}$ GHz. Several experiments have been carried out to test the Sband prototype and assess its performance against larger drones and cars. Results have shown targets could be successfully detected and their micro-Doppler signatures extracted using Short-Time Fourier Transform (STFT) techniques.
\end{abstract}

Keywords - FMCW Radar Development, Micro-Doppler Extraction, Micro-UAV, Nano-Drones, Insects.

\section{INTRODUCTION}

Nano-targets, such as nano-drones and insects, present a detection challenge to conventional radar because they are tiny and fly at a relatively low speed. Yet, if detected they present characteristics that could enable radar classification by their micro-Doppler signatures. This distinctive Doppler pattern is induced by any form of micro-motion of rotating or flapping components that cause alteration in the frequency domain and provide a unique target signature [1]. Indeed, micro-Doppler has been proven a potential technique that could cope well with this type of challenge for larger drones [2] [3]. Previous research has investigated micro-UAV (larger than $20 \mathrm{~cm}$ ) detection by using L, S and X-band digital array radar [4] [5]. The targets used in [5], for example, were a Phantom, a Mavic Pro and a Mavic Air with a blade size of 24 $\mathrm{cm}, 21 \mathrm{~cm}$ and $13.5 \mathrm{~cm}$, respectively. In [6], classification performance of different types of mini-UAVs (with an average size of about $1 \mathrm{~m}$ ) was improved with deep learning techniques. Recent developments in the field of nano-size drones [7] [8] are likely to result in a need to design and develop sensors which could detect much smaller threats (i.e. smaller than $5 \mathrm{~cm}$ ). To the best of our knowledge, there is very little, if any, in the literature investigating detection and classification of individual insect-size drones.

On the other hand, there has been a significant amount of research focusing on crop protection from insect pests [9][11]. In particular, entomologists have employed a variety of strategies to minimise chemical inputs without reducing agricultural output. Advancing studies on insect migration aim to improve outbreaks management especially swarm insect infestation that can cause devastating consequences. Insects continuously migrate vast distances foraging for food, breeding and to shelter. Entomologists have historically used X-band Vertical Looking Radar (VLR) and Harmonic Radar (HR) to monitor long-range and short-range insect movement, respectively. VLR observe insects at altitudes between $150 \mathrm{~m}$ to $1.2 \mathrm{~km}$ above the ground level [12]. The limitation with VLR is that being designed as marine pulsed radar systems they do not provide coverage below $150 \mathrm{~m}$ and high range resolutions [13]. Hence, altitudes below $150 \mathrm{~m}$ have been neglected and entomologists have had to rely primarily on swarm detections rather than detection of individual insects. HR relies on a transponder that is mounted on the insect and therefore can only help in behavioural studies investigating short-range low-flying insect [14]. The authors of [15] have also acknowledged that conventional HR still use big transponders which may affect the insect behaviour. Needless to say, it is more appropriate to have contactless detection. The authors of [16] claimed that the use of micro-Doppler with Wband FMCW radar is a reliable approach in insect observation.

Frequency Modulated Continuous Wave (FMCW) radar is one of the candidate technologies to address these technical challenges. FMCW radar continuously transmit and receive signals and therefore can provide full surveillance coverage without blind range. In addition to this, they are relatively low cost and still provide Doppler information to allow the extraction of micro-Doppler signatures for target classification. The goal of this research is to build a low-cost 
FMCW radar at $24 \mathrm{GHz}$ and this paper presents the results of a first intermediate research step during which an S-band FMCW radar prototype has been developed to assess design performance and inform the next research phases at higher frequencies. The main reason S-band was selected for the intermediate prototype is because of availability of very lowcost off-the-shelf components at this range of frequencies. Experiments have been carried out with a car and larger drones at S-band and results have proved that targets can be detected and their micro-Doppler signatures extracted.

\section{THEORETICAL DESCRIPTION}

FMCW radar transmit a sawtooth waveform consisting of periodic Linear Frequency Modulated (LFM) ramps

$$
S_{T}=\cos \left(2 \pi f_{o} t+\gamma \pi t^{2}\right)
$$

With chirp rate

$$
\gamma=B / T_{r}
$$

where $B$ is the signal bandwidth over the ramp duration $T_{r}$ and $f_{o}$ is the carrier frequency. A target at a range $R_{0}$ will produce an echo

$$
S_{R}=\cos \left(2 \pi f_{o}\left(t-t_{o}\right)+\gamma \pi\left(t-t_{o}\right)^{2}\right)
$$

with a time delay $t_{0}=2 R_{0} / c$, where $c$ is the speed of propagation. A double balanced mixer is used to beat the received signal $S_{R}$ with a copy of the transmitted signal $S_{T}$ and provide an output

$$
\begin{aligned}
z(t)=S_{T} \cdot S_{R}= & \left\{\cos \left(2 \pi f_{o} t_{o}+2 \pi \gamma t_{o} t-\pi \gamma t_{o}{ }^{2}\right)\right. \\
& +\cos \left(4 \pi f_{o} t+2 \pi \gamma t^{2}-2 \pi f_{o} t_{o}\right. \\
& \left.\left.-2 \pi \gamma t_{o} t+\pi \gamma t_{o}{ }^{2}\right)\right\}
\end{aligned}
$$

which is low-passed filtered to obtain the final signal

$$
y(t)=\cos \left(2 \pi f_{0} t_{0}+2 \pi \gamma t_{o} t-\pi \gamma t_{0}^{2}\right)
$$

of frequency $\gamma t_{o}$. The frequency of the resulting signal is called the beat frequency and is equal to the product between the chirp rate and the time delay. By measuring the beat frequency the radar calculates the time delay and provides an estimation of the range of the target. Since a double balanced mixer is used and because the output is low-pass filtered to discard the high-frequency components, the output is characterised by a symmetrical frequency spectrum consisting of two components as in Fig 1.

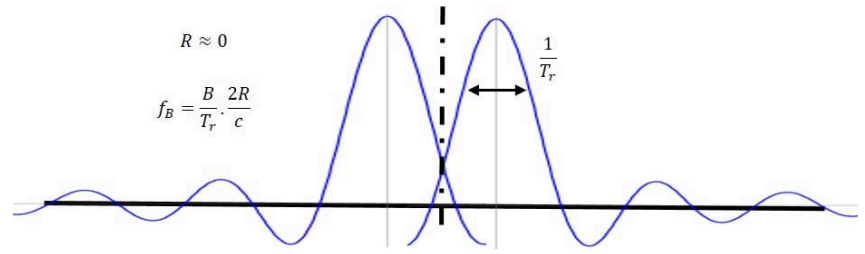

Fig 1: Overlapping Spectrums

Using an IQ demodulator will provide only the positive spectrum component but at a higher prototyping cost. Without the IQ demodulator, the radar parameters are selected so that the two spectrums are the farthest away to each other and do not overlap. A compromise needs to be found noting that increasing the ramp duration, results in narrower lobes but, for a fixed bandwidth, reduces the frequency $f_{b}$. Increasing the chirp bandwidth, reduces the overlapping range of the radar and the range resolution. Equation (6) shows the relationship between the radar parameters that affect the beat frequency.

$$
f_{b}=\frac{B}{T_{r}} \cdot \frac{2 R_{0}}{c}
$$

The IF signal is digitised and segmented into small chunked windows of the length of the ramp duration. Then, an FFT is applied to each window to obtain a range profile. The following step involves a second FFT along each range to provide a range-Doppler map. This will inherently show a peak return at the target Doppler and distance. The Doppler of the target can be extracted after zero-Doppler removal in each row of the 2D range-Doppler map (RDM). Once the peak corresponding to the target is identified, the slow time target returns are segmented into small windows and another FFT is applied to the slow time data to obtain the target micro-Doppler signature.

\section{PROTOTYPE IMPLEMENTATION}

A sawtooth chirp waveform is generated with an Anritsu MS2691A as in Fig 2 with a carrier frequency of $3.5 \mathrm{GHz}$. Due to the limitation of the signal generator, the maximum signal bandwidth is $50 \mathrm{MHz}$ corresponding to a range resolution of $3 \mathrm{~m}$. Each ramp spans from $3.5 \mathrm{GHz}$ to $3.55 \mathrm{GHz}$ with a duration of $1 \mathrm{~ms}$, leading to a Doppler bandwidth of 1 $\mathrm{kHz}$.

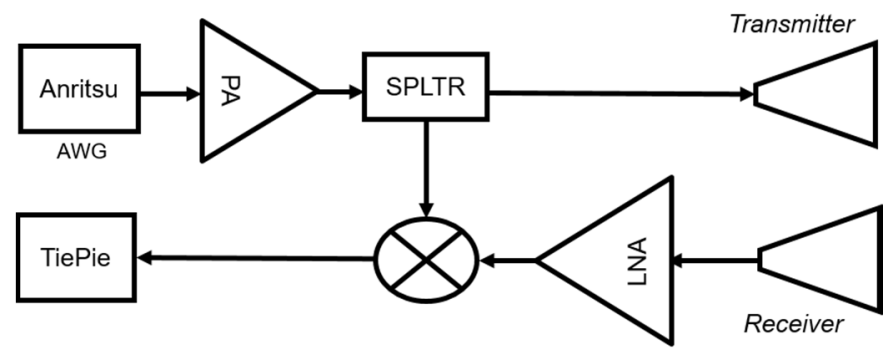

Fig 2: Block Diagram of FMCW Radar Prototype 
The S-band FMCW radar prototype consists of two components in the transmission chain and two components in the receiving chain. In transmission, a mini-circuits ZX60$362 \mathrm{GLN}-\mathrm{S}+$ amplifies the signal from the Anritsu generator before splitting it with a mini-circuits ZX10-2-622+ into two signals, one directed to the transmit antenna and the other one directed to the receiver to provide a copy of the transmit signal which is fed to the Local Oscillator (LO) port of the mixer. The transmit and receive antennas are identical Broadband Horn Antenna LB-7180 with a gain of $12 \mathrm{dBi}$, length $244 \mathrm{~mm}$ and aperture $160 \times 230 \mathrm{~mm}$. The reception part comprises a mini-circuits ZX05-C42-S+ double balanced mixer and a low-noise amplifier ZX60-362GLN-S+ which is used in order to increase the strength of the signal before digitising the signal with a TiePie HS5 USB oscilloscope. Fig 3 shows the RF components of the radar. The power output is about $+13 \mathrm{dBm}$.

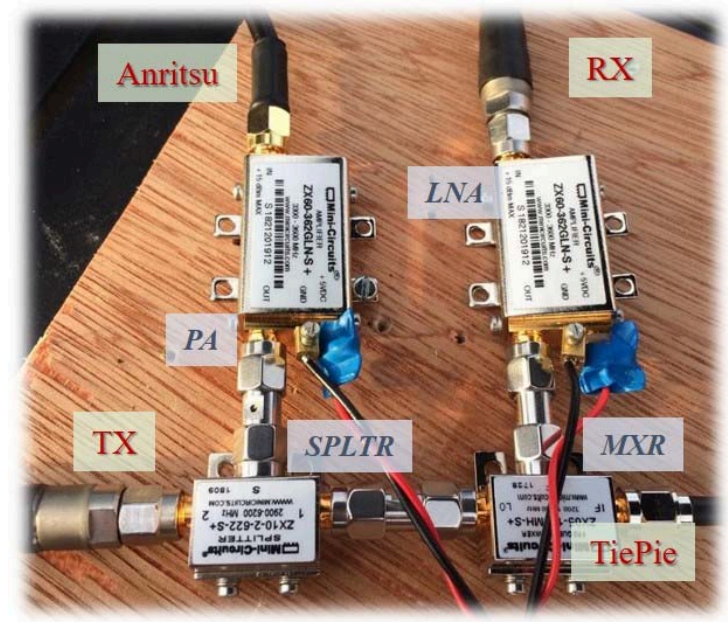

Fig 3: A Close-Up of Radar Prototype

\section{EXPERIMENTAL RESULTS \& DISCUSSION}

Two experiments were carried out to test the prototype. The goal of the first experiment was to assess the radar response to the Doppler shift of a single moving car and the goal of the second experiment was to demonstrate the prototype could detect the micro-Doppler signature induced by three different targets with propeller blades.
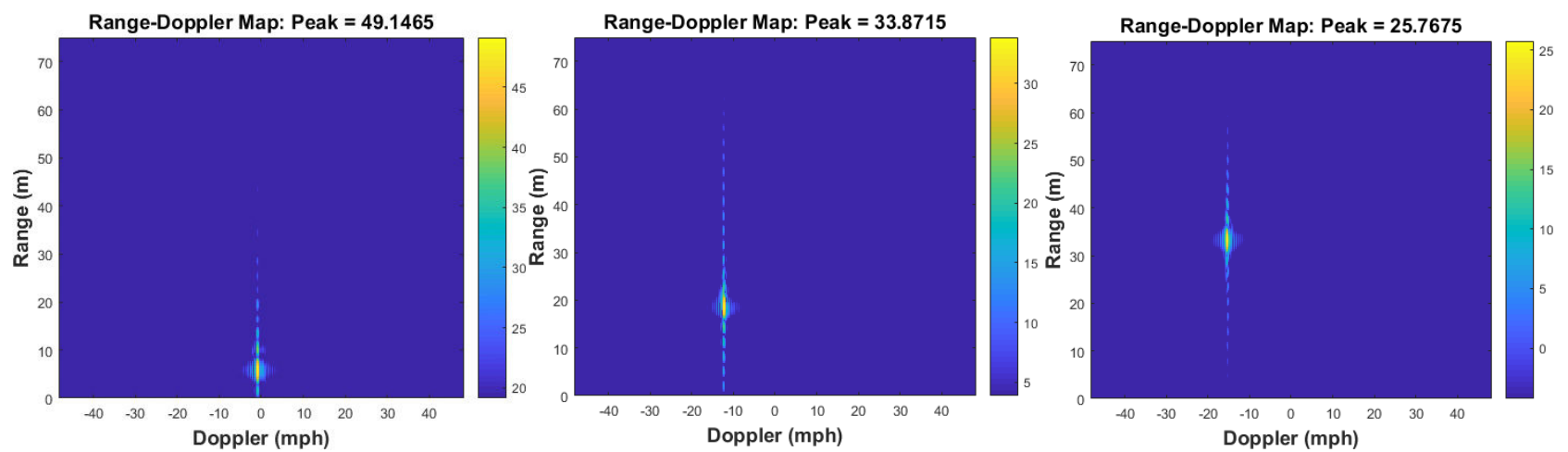

Fig 5: Experimental Results of a Target Moving Away from Radar 

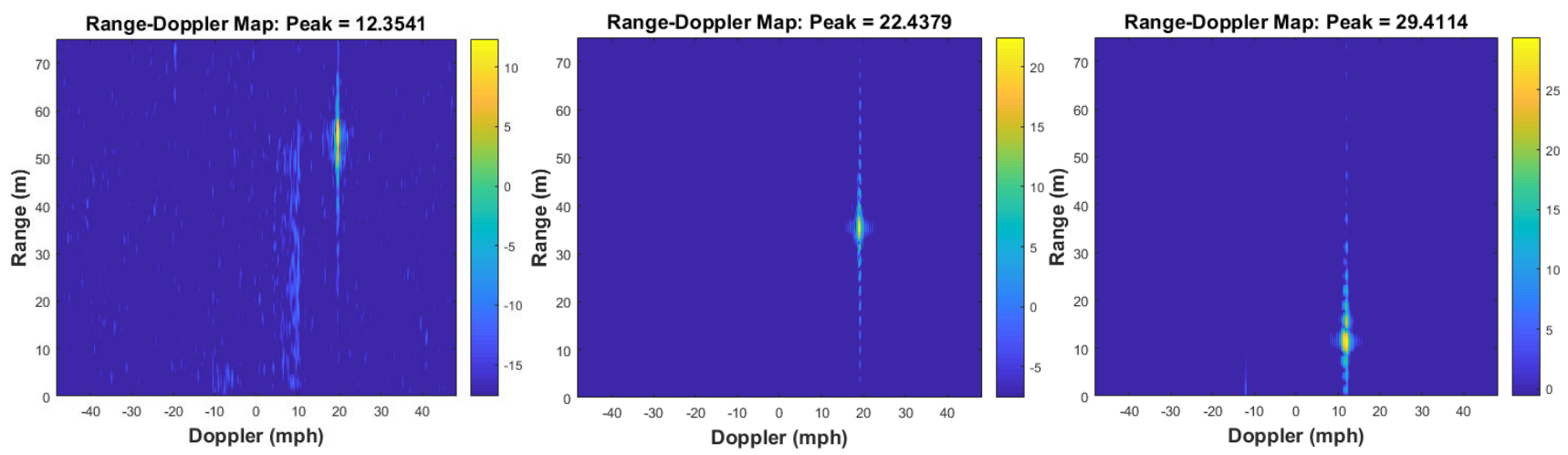

Fig 6: Experimental Results of a Target Moving Towards the Radar

\section{B. Propeller Observation}

The second experiment was run to assess the ability of the prototype to detect the micro-Doppler signatures of different types of targets with propeller blades. A photo of the three targets is shown in Fig 7. The data was collected with the Sband radar prototype and the results analysed. Results obtained for the three targets are outlined below.

(a) One-blade propeller. The target consists of a $46 \mathrm{~cm}$ long blade rotating at approximately 120 rotations per minute (rpm), corresponding to an angular speed of about $2 \mathrm{~Hz}$, and leading to a tangential velocity of about $5.78 \mathrm{~m} / \mathrm{s}$. During the measurement, the rotation of the blade was perpendicular to the Line Of Sight (LOS) of the radar in order to induce the maximum Doppler response from the target. A metallic sphere was attached at the tip of the blade to increase the SNR and investigate the extraction of the micro-Doppler signature. Fig 8(a) and Fig 9(a) show the RDM and micro-Doppler of the target. It is noticeable that there is periodical line in the $\mathrm{RDM}$ plot with the separation between the two lines is $2 \mathrm{~Hz}$. This represents the rate rotation of the blade. A periodical sinusoidal is also visible in the micro-Doppler plot with 2 complete cycle within $1 \mathrm{~s}$ and the result show that the maximum Doppler frequency achievable is $136 \mathrm{~Hz}$. Data was digitized with a sampling frequency of $100 \mathrm{kHz}$ and 100 kiloSamples were collected to obtain measurements of $1 \mathrm{~s}$ integration time. The window length used to extract the micro-Doppler signature was $18 \mathrm{~ms}$.

(b) Remote Control Helicopter. The target with $18.5 \mathrm{~cm}$ long blades was placed on a stand with the blade rotating in a stationary position. The sampling frequency was $100 \mathrm{kHz}$ and $100 \mathrm{kSamples}$ were collected (that is $1 \mathrm{~s}$ signatures). Fig 8(b) shows the RDM of the target. Results show the spectrum consists of lines separated of about $20 \mathrm{~Hz}$. The window length used to extract the micro-Doppler signature was $13 \mathrm{~ms}$.
Results in Fig 9(b) show the micro-Doppler of the target presents about 20 blade flashes within $1 \mathrm{~s}$ long corresponding to a rotational speed, of each blade, of about $600 \mathrm{rpm}(10 \mathrm{~Hz})$. The maximum Doppler frequency is approximately $273.5 \mathrm{~Hz}$ corresponding to a tangential velocity of $11.6 \mathrm{~m} / \mathrm{s}$. The SNR for this scenario is weaker compared to the previous case since it deals with a lower RCS target. Doppler results also indicate a strong vibrations from the body of the target and the stand with stronger Doppler returns around $0 \mathrm{~Hz}$.

(c) Hexacopter drone. The third target is characterised by twelve $10 \mathrm{~cm}$ long propeller blades. Similar to the previous case, the drone was placed on a stand during the measurements so that the blades could rotate in a stationary position. The sampling rate was $50 \mathrm{kHz}$ and $100 \mathrm{kSamples}$ resulting in a dwell time of $2 \mathrm{~s}$. Fig 8(c) shows the RDM of the target and it is shown that the SNR signal is weakest compare to the previous two as the size of the blade decrease making the signal return from the blade propellers are weaker even so the rotational speed of hexacopter is faster than the others. The separation of each line is approximately $65 \mathrm{~Hz}$. The window length used to extract the micro-Doppler signature shown in Fig 9(c) was 25 ms. Results show several lines in the micro-Doppler plot which indicate a much faster rotation speed than the previous targets.

Results corroborate that the faster the rotational blade, the higher is the separation between lines in the RDM plot. Because the chirp duration $T_{r}$ will determine the span of the Doppler axis in RDM and STFT plot $\left(f_{\text {span }}= \pm 1 / T_{r}\right)$. Equation (8) indicates the minimum ramp duration required in order to avoid ambiguous Doppler measurements which depends on the wavelength $\lambda$ of the radar, the rotational rate of the blade $f_{\text {rot }}$ and blade length $L$. With a constant $\lambda$ and $L$, with higher $f_{\text {rot }}$ demands a smaller $T_{r}$.

$$
T_{r_{\text {min }}}=\lambda /\left(8 \pi f_{\text {rot }} L\right)
$$




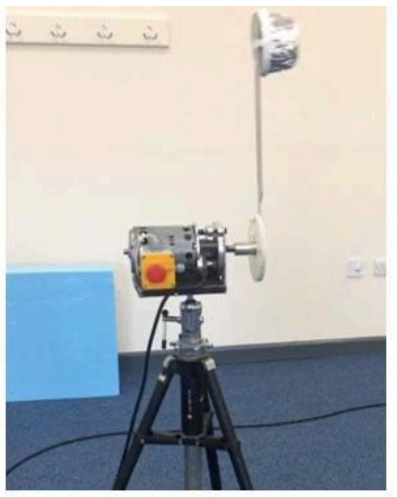

(a)

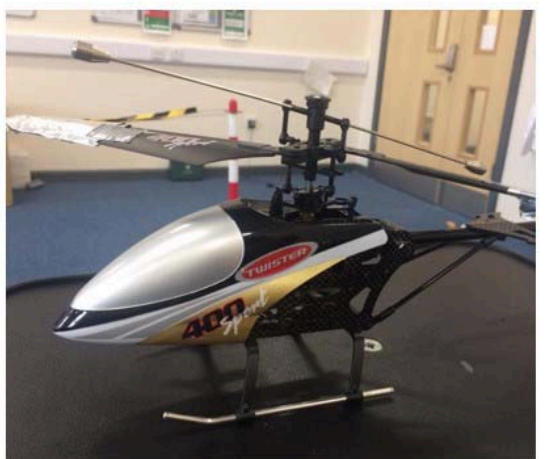

(b)

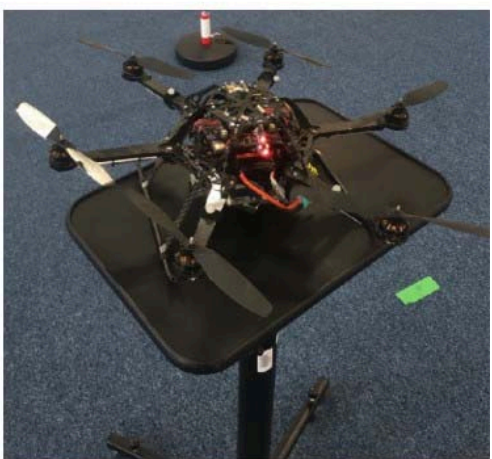

(c)

Fig 7: Different Radar Targets (a) One-Blade Propeller, (b) RC Helicopter (c) Hexacopter

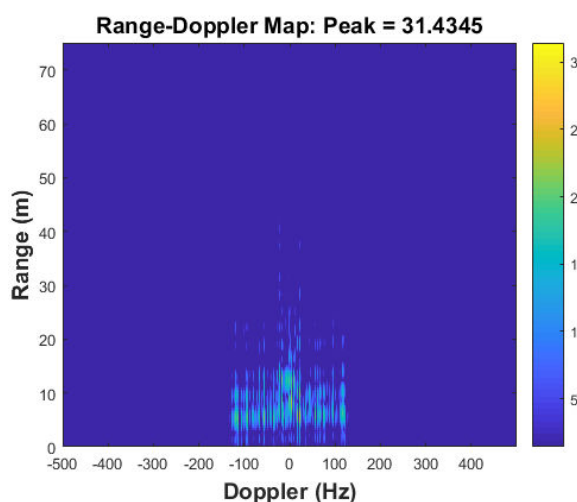

(a)

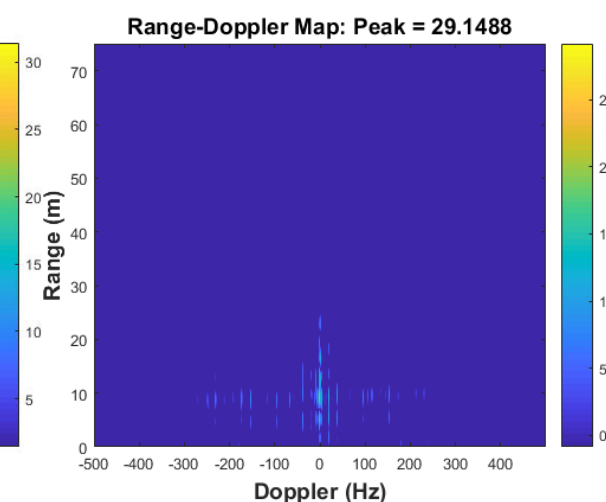

(b)

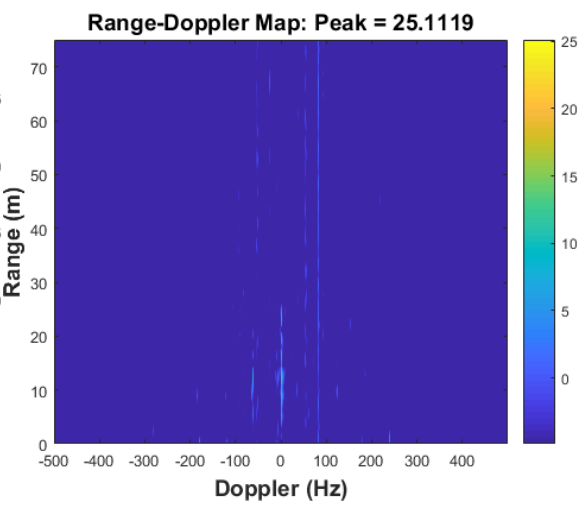

(c)

Fig 8: Range Doppler Map of (a) One-Blade Propeller, (b) RC Helicopter and (c) Hexacopter

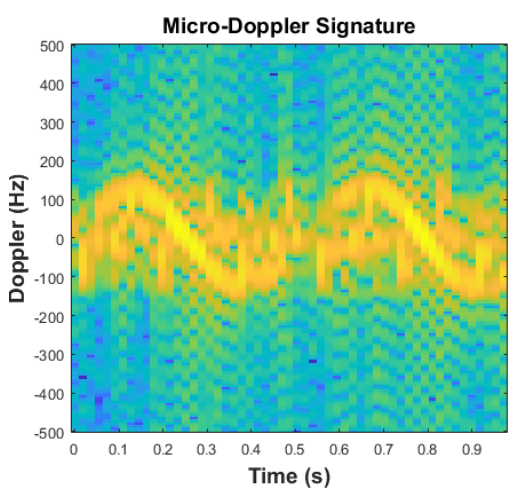

(a)

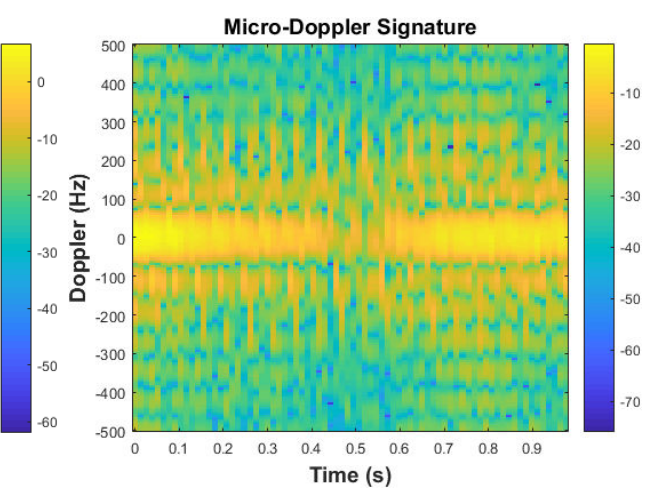

(b)

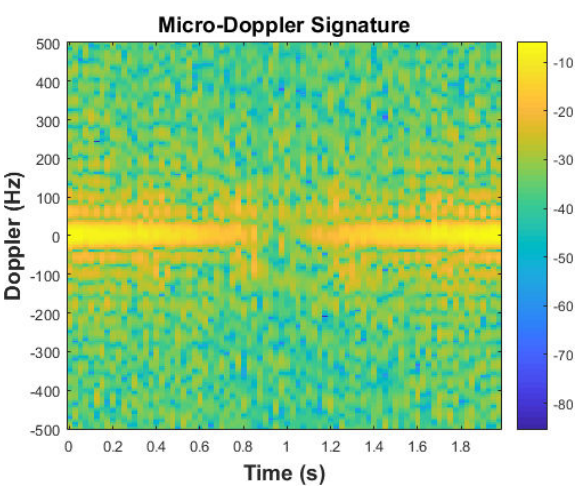

(c)

Fig 9: Micro-Doppler Signature of (a) One-Blade Propeller, (b) RC Helicopter and (c) Hexacopter 


\section{CONCLUSION}

The purpose of the present research is to build a low-cost FMCW radar at $24 \mathrm{GHz}$ for nano-targets detection. A S-band radar prototype was initially designed, developed and evaluated the robustness of the FMCW radar prototype. It is served as a base structure to work on a higher frequency that could potentially increase the signal energy returned in dealing with a lower RCS and make the nano-target detection become possible. The data was collected then analysed with a detailed post-processing technique using an STFT algorithm to detect the Doppler shift and to extract the microDoppler signature of the taargets. The study suggests that the target characteristics is possible regardless the size of the target by observing the range-Doppler map as well as microDoppler signature. Future work is already underway by the authors.

\section{ACKNOWLEDGMENT}

The authors would like to thank the School of Aerospace Engineering, Universiti Sains Malaysia, Malaysia and the Malaysian Ministry of Higher Education for sponsoring the PhD studies of Safiah Zulkifli at Cranfield University.

\section{REFERENCES}

[1] V. C. Chen, The Micro-Doppler Effect in Radar, Artech House, 2011.

[2] S. Rahman., D.A. Robertson, "Radar micro-Doppler signatures of drones and birds at K-band and W-band," Sci Rep 8, 17396, 2018.

[3] S. Rahman and D. Robertson, "Time-Frequency Analysis of MillimeterWave Radar Mi-cro-Doppler Data From Small UAVs," 2017 Sensor Signal Processing for Defence Conference (SSPD), London, 2017, pp. $1-5$

[4] A. Aldowesh, T. Alnuaim and A. Alzogaiby, "Slow-Moving MicroUAV detection with a small scale Digital Array Radar," 2019 IEEE Radar Conference (RadarConf), Boston, MA, USA, 2019, pp. 1-5.
[5] A. Aldowesh, T. BinKhamis, T. Alnuaim and A. Alzogaiby, "Low Power Digital Array Radar for Drone Detection and Micro-Doppler Classification," 2019 Signal Processing Symposium (SPSympo), Krakow, Poland, 2019, pp. 203-206.

[6] A. Huizing, M. Heiligers, B. Dekker, J. de Wit, L. Cifola and R. Harmanny, "Deep Learning for Classification of Mini-UAVs Using Micro-Doppler Spectrograms in Cognitive Radar," in IEEE Aerospace and Electronic Systems Magazine, vol. 34, no. 11, pp. 46-56, 1 Nov. 2019

[7] M. Longhi and G. Marrocco, "Flying sensors: Merging Nano-UAV with radiofrequency identification," 2017 IEEE International Conference on RFID Technology \& Application (RFID-TA), Warsaw, 2017, pp. 164168.

[8] E. Tsykunov, L. Labazanova, A. Tleugazy and D. Tsetserukou, "SwarmTouch: tactile interaction of human with impedance controlled swarm of nano-quadrotors," 2018 IEEE/RSJ International Conference on Intelligent Robots and Systems (IROS), Madrid, 2018, pp. 4204-4209.

[9] J. Baum, T. Bogaert, W. Clinton, G. Heck, P. Feldmann, et al. "Control of coleopteran insect pests through RNA interference," Nat Biotechnol 25, 1322-1326, 2007.

[10]K. Gordon and P. Waterhouse, "RNAi for insect-proof plants," Nat Biotechnol 25, 1231-1232, 2007.

[11]D. Boddupally, S. Tamirisa, S. R. Gundra, D. R. Vudem and V. R. Khareedu, "Expression of hybrid fusion protein (CrylAc::ASAL) in transgenic rice plants imparts resistance against multiple insect pests," Scientific Reports 8, no. 8458, 2018.

[12]V. A. Drake and D. R. Reynolds, Radar Entomology Observing Insect Flight and Migration, CABI, 2012

[13]C. Hu, W. Li, R. Wang, T. Zhang and W. Li, "Insect speed extraction method based on a high resolution and full polarisation radar with vertical-looking mode," in The Journal of Engineering, vol. 2019, no. 19, pp. 5889-5892, 102019.

[14]N. Tahir and G. Brooker, "The investigation of millimetre wave optical harmonic transponders and radar for monitoring small insects," 2013 IEEE Topical Conference on Wireless Sensors and Sensor Networks (WiSNet), Austin, TX, 2013, pp. 22-24.

[15]Y. T. Liu, M. L. Hsu, H. Wang and Z. M. Tsai, "A differential miniature transponder for 9.4/18.8 GHz harmonic bee searching radar with low gain degradation from bee's body," 2016 IEEE MTT-S International Microwave Symposium (IMS), San Francisco, CA, 2016, pp. 1-4.

[16]R. Wang, C. Hu, X. Fu, T. Long, and T. Zeng, "Micro-Doppler measurement of insect wing-beat frequencies with W-band coherent radar,” Scientific Reports 7, no. 1396, 2017. 